\title{
Removal of a broken intramedullary femoral nail with an unusual pattern of breakage: a case report
}

\author{
Juan Pretell Mazzini · Juan Rodriguez Martin • \\ Carlos Resines Erasun
}

Received: 29 July 2008/Accepted: 13 August 2009/Published online: 24 September 2009

(C) Springer-Verlag 2009

\begin{abstract}
To the best of our knowledge, only 3 cases, including the present case, have been reported with a three part broken pattern. However, this is the first case associated with a distal locking screw broken. We report the case of a 31-year-old patient who sustained an open femoral shaft fracture. The fracture was stabilized with a Kuntcher femoral nail. After 7 months of the initial surgery he presented with a three part broken intramedullary nail and the distal locking screw broken. We used a combined technique for the removal of the nail through the nonunion fracture site; we used a pull out technique for the middle fragment and a curved thin hook for the distal fragment. Then we applied bone allograft and stabilized with a cannulated intramedullary femoral nail (Synthes, Oberdorf, Switzerland). After 2 years of follow up the nonunion was consolidated and the patient presented a good clinical outcome. This is of particular interest because it is a unique case and the association with a broken distal locking screw is reported for the first time in this study. A combination of methods through the nonunion site approach and an alternative instrumental is a good method for the removal of a hollow femoral intramedullary nail with this unusual pattern of breakage.
\end{abstract}

Keywords Broken nail - Intramedullary nailing ·

Removal · Narrow hollow · Guide wire

J. Pretell Mazzini $(\bowtie) \cdot J$. Rodriguez Martin .

C. Resines Erasun

Hospital Doce de Octubre, Avd de Córdoba s/n,

28041 Madrid, Spain

e-mail: el_giova23@yahoo.com

\section{Introduction}

Intramedullary nails are commonly used to treat fractures of the femoral shaft [1-4]. The method has the advantage of requiring only small surgical incisions and achieving stable reduction without opening the fracture, with the load being carried physiologically down the mechanical axis of the bone [5].

As the indications for nailing have expanded, the rate of mechanical failure has also increased [2, 6]. An intramedullary nail subjected to cyclical loading may develop fatigue failure if the stresses on the nail exceed its endurance limit [7]; this is a well-recognised complication [8].

This can be influenced by a large number of factors, including fracture instability, slow progress to union, an inappropriate rehabilitation program, the mechanical characteristics of the nail, or the primary surgical technique $[5,7,9,10]$. The result is that the nail breaks, and removal of the fragments is not always a simple procedure $[11,12]$.

We can find only two previous reports similar to this case; it is of interest because the nail removal was performed using the non-union site as the entry portal for the removal of the middle and distal fragments and the insertion of bone graft, thereby avoiding opening the knee joint.

\section{Case report}

A 31-year-old man sustained a Gustilo I open fracture of the left femoral shaft at the junction of the middle and distal thirds as a result of a motorcycle accident. The fracture was classified A3 according to the AO system (Fig. 1a, b). There were no other injuries.

It was stabilised with an 11 by $400 \mathrm{~mm}$ intramedullary Küntscher femoral nail after closed reaming to $12 \mathrm{~mm}$, 
Fig. 1 Femoral shaft fracture. a Plain radiograph anteroposterior view. b Plain radiograph lateral view
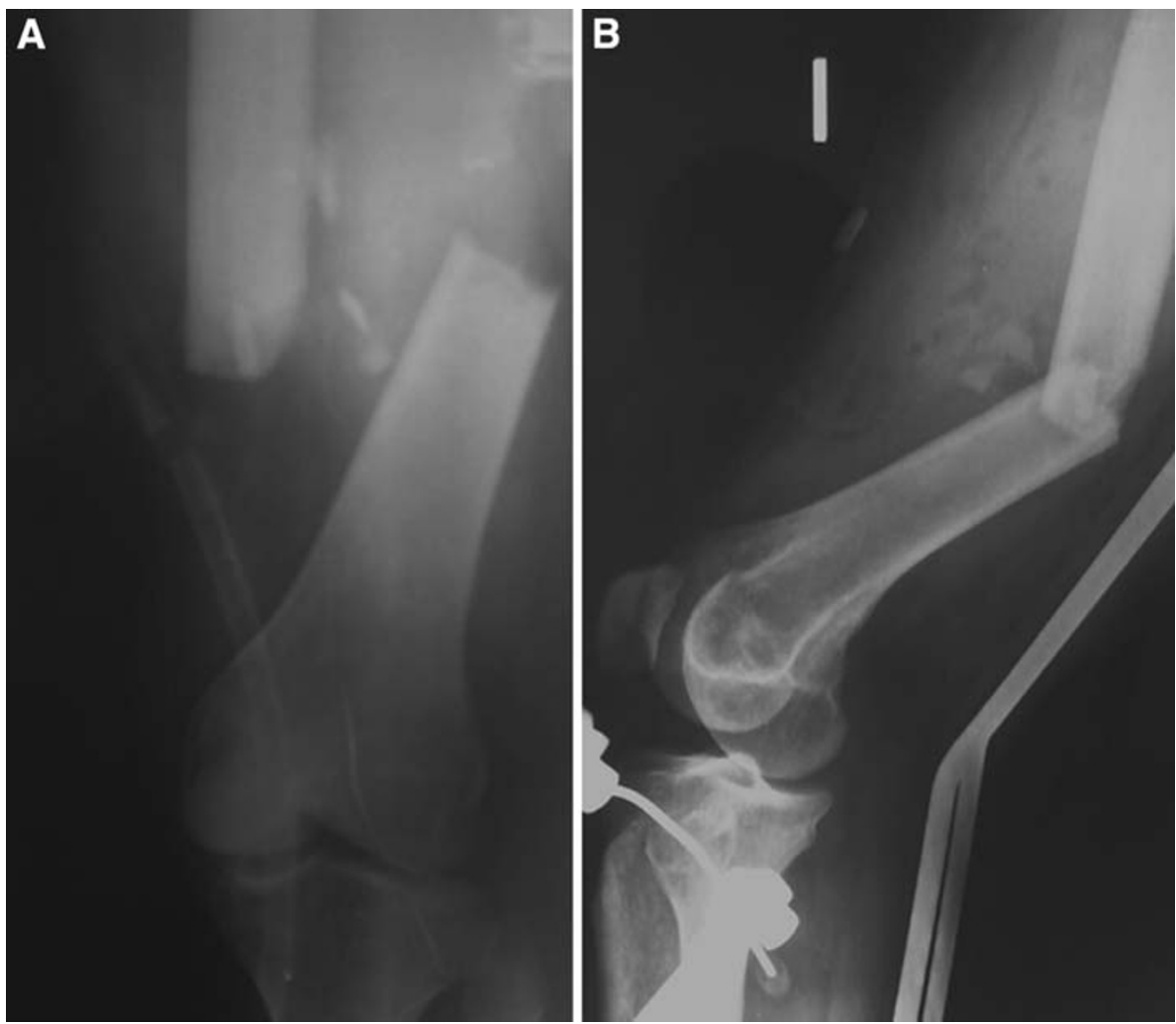
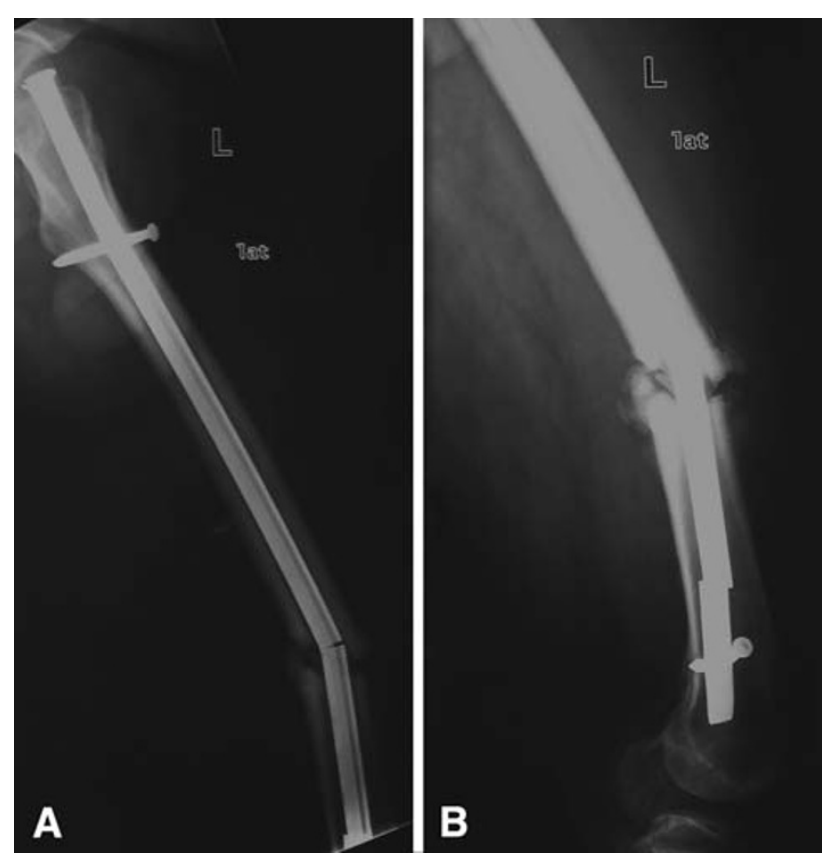

Fig. 2 Three part broken femoral Küntscher nail with broken distal locking screw. a Nail breakage at the non-union site. b Second nail breakage proximal to the distal locking hole with a broken locking screw

locked proximally and distally with one screw. A Friedrich technique was performed at the fracture site leaving a Penrose drain in situ.
There were no postoperative complications. The patient was allowed to walk with crutches, partially weight bearing initially, increasing to full as tolerated; he also followed a rehabilitation program.

Four months after the initial surgery he presented with diffuse knee pain without any history of trauma; X-ray showed breakage of the distal screw with some callus formation so we decided to leave it to dynamise the fracture site.

Three months later he presented to the emergency room with acute onset of severe pain and inability to weight bear. Plain radiographs revealed a femoral non-union and a three part broken nail (Fig. 2a, b). There was no evidence of infection.

For the revision surgery (exchange nailing and bone grafting) the patient was placed in the supine position on a fracture table under image intensifier control. The proximal nail fragment was removed using a standard technique through the original entry point. The two parts of the distal broken screw were removed through lateral and medial incisions using a grasping device.

The non-union site was then opened laterally, taking tissue biopsy for culture and preparing the fracture site for grafting. The medullary cavity was exposed and the middle nail fragment removed with a cement rongeur from the Moreland ${ }^{\circledR}$ cemented hip revision instrumentation (DePuy, Warsaw IN, USA), by grasping the proximal end and removing it through the non-union site (Fig. 3a). 
Fig. 3 Diagram of the pull out technique for the removal of the distal fragments. a The fracture was flexed a little to expose the implant and facilitate gripping the proximal end with the cement rongeur. b Introduction of the curved thin hook through the femoral and nail canal into the nail tip for the removal of the distal fragment. c

Manoeuvres made with the curved thin hook for the removal of the distal fragment

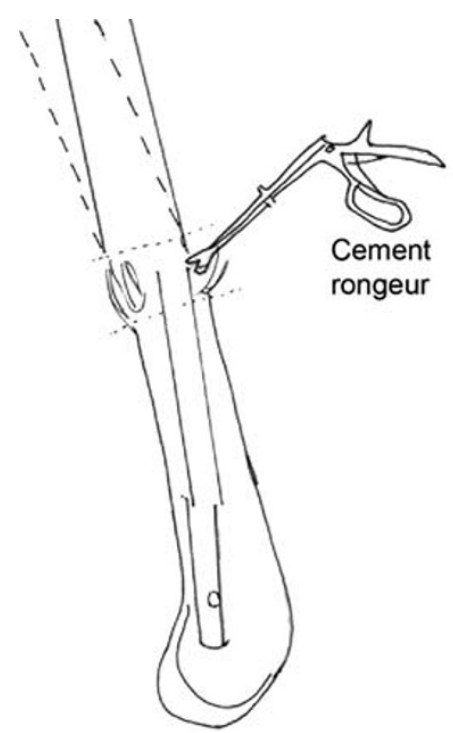

A

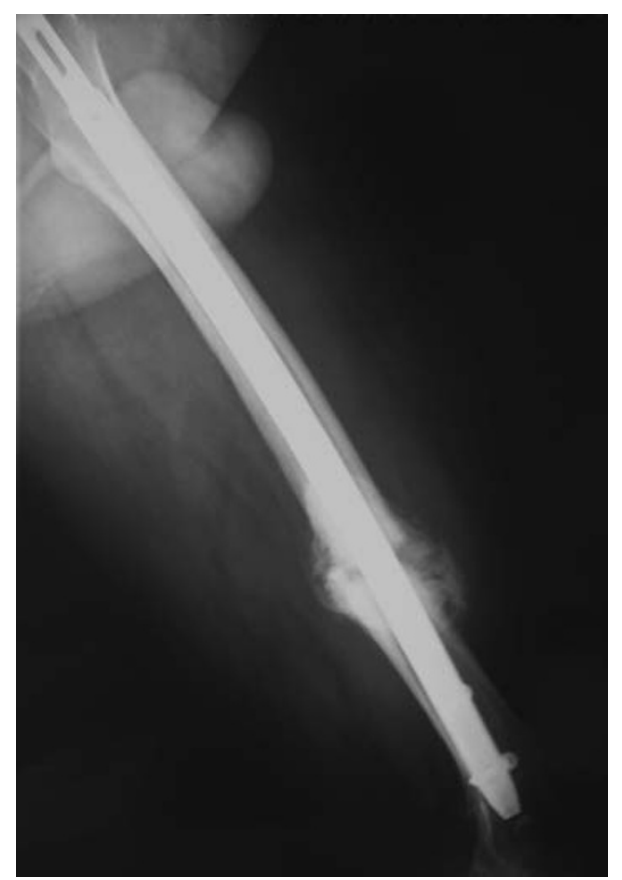

Fig. 4 Four months postoperative axial view showing good callus formation

For the remaining distal nail fragment, we initially passed an olive-tipped guide wire through the non-union site down the nail canal, but the nail tip was too wide and this attempt failed. We then used a curved thin hook from the same Moreland ${ }^{\circledR}$ hip revision instrumentation, passing it through the nail canal, engaging the nail tip, and removing the final nail fragment through the non-union site (Fig. 3b, c).

After antegrade reaming to $16 \mathrm{~mm}$, a $15 \times 380 \mathrm{~mm}$ cannulated femoral nail (Synthes ${ }^{\circledR}$, Oberdorf, Switzerland)
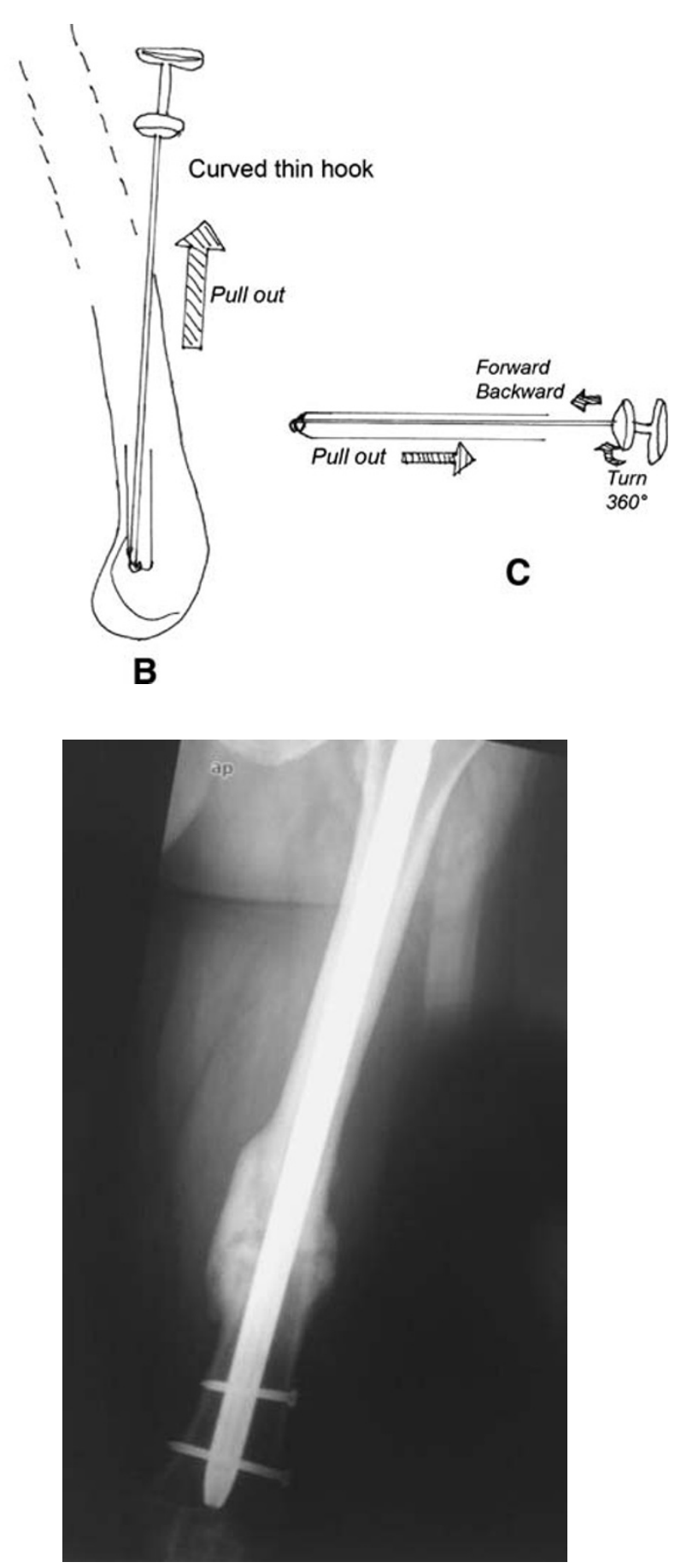

Fig. $5 \mathrm{AP}$ view at 2 years

was inserted with two distal locking screws (Fig. 4). Allograft bone was used to fill the non-union area. The operating time was $75 \mathrm{~min}$.

There were no postoperative complications. One month later the patient was allowed partial weight bearing progressing to full as tolerated. At 2-years follow up the fracture was united (Fig. 5) and the patient was walking fully weight bearing; knee range of motion was $0-110^{\circ}$ (Fig. 6a, b). 
Fig. 6 Clinical outcome. a Knee extension. b Knee flexion
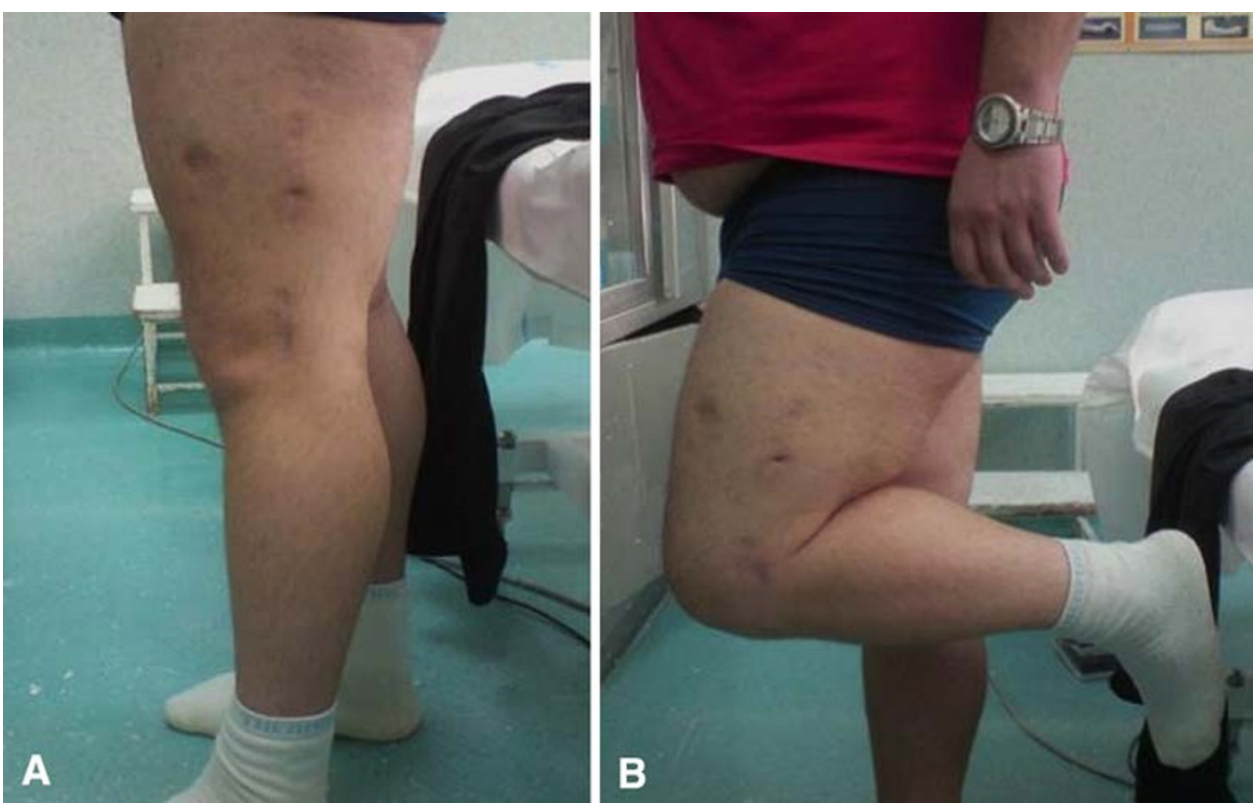

\section{Discussion}

Several studies have shown a significant prevalence of implant failure associated with femoral fractures distal to the isthmus [7, 13], with an incidence between $1 \%$ and $3.3 \%[8,13]$.

Multiple techniques for broken nail removal have been described $[5,8,12]$ and retrieval of the distal fragments without destructive surgery [5] remains a challenging problem with no universally accepted technique [12].

To our knowledge there are only two recorded cases with a similar pattern of breakage. Sivananthan et al. [12] reported 12 broken implants which included one broken into three parts in a similar pattern; this was removed with a pull out technique using a $3 \mathrm{~mm}$ smaller nail impacted into the distal fragment. Kouvidis et al. [8] reported a three part broken G-K femoral nail; he used a push out technique through a transarticular intercondylar approach. In both cases the nails were hollow and the distal locking screws were not broken.

There are some peculiarities in our case that make it unique: the distal locking screw was broken, and this increased the general difficulty because there was other hardware to be removed and the techniques described by Sivananthan [12] and Levy [10] (impacting a smaller nail and using a pull out technique) were not possible because there was nothing to counteract an impacting force.

Sivananthan et al. [12] used a proximal approach to remove both distal fragments. We could not because the two distal fragments were slightly displaced. A transarticular approach would have been an unnecessary additional injury to the patient, because the distal fragment was not tight and could be removed without difficulty.

\section{Conclusion}

The removal of a nail with this type of breakage through the fracture site is a good option when bone allograft is to be used. Combined techniques of removal are sometimes required for unusual patterns of nails breakage.

Acknowledgments The authors appreciate the contribution of Dr. A. Diaz Martin for the literature search used to elaborate this manuscript.

\section{References}

1. Khan FA (1992) Retrieval of a broken intramedullary femoral nail. Injury 23(2):129-130

2. Marwan M, Ibrahim M (1999) Simple method for retrieval of the distal segment of the broken interlocking intramedullary nail. Injury 30(5):333-335

3. Browner BD (1986) Pitfalls, errors and complications in the use of the locking Küntscher nail. Clin Orthop 212:192-208

4. Albert JS (1996) Fracture of the femoral shaft. Surgery 14:102117

5. Giannoudis PV, Matthews SJ, Smith RM (2001) Removal of the retained fragment of broken solid nails by the intramedullary route. Injury 32:407-410

6. Park SY, Yang KH, Yoo JH (2006) Removal of a broken intramedullary nail with a narrow hollow. J Orthop Trauma 20(7):492-494

7. Krettek C, Schandelmaier P, Tscherne H (1997) Removal of a broken solid femoral nail: a simple push-out technique. A case report. J Bone Joint Surg Am 79(2):247-251

8. Kouvidis GK, Galanakis IA, Giannoudis PV, Hadjipavlou AG (2008) Three-part broken intramedullary nail reconsideration: a case report and review of the literature. J Trauma (Epub ahead of print)

9. Brewster NT, Ashcroft GP, Scotland TR (1995) Extraction of broken intramedullary nails-an improvement in technique. Injury 26:286 
10. Levy O, Amit Y, Velkes S, Horoszowski H (1994) Brief reports: a simple method for removal of a fractured intramedullary nail. $\mathrm{J}$ Bone Joint Surg Br 76:502

11. Wise DJ, Hutchins PM (1996) Novel method for removal of a broken GK femoral. Injury 27:294-295
12. Sivananthan KS, Raveendran K, Kumar T, Sivananthan S (2000) A simple method for removal of a broken intramedullary nail. Injury 31(6):433-434

13. Franklin JL, Winquist RA, Benirschke SK, Hansen ST Jr (1988) Broken intramedullary nails. J Bone Joint Surg Am 70:1463-1467 